\title{
Capacidade funcional de idosos atendidos pelo programa saúde da família em Benevides, Brasil
}

\section{Functional capacity of the elderly cared by the family health program in Benevides, Brazil}

\author{
Capacidad funcional de adultos mayores atendidos por el programa \\ de salud de la familia en Benevides, Brasil
}

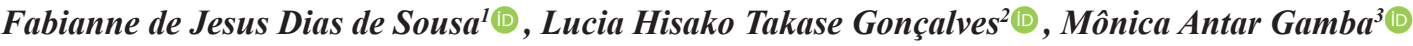

\section{Histórico}

Recibido:

04 de febrero de 2018

Aceptado:

10 de abril de 2018

1 Doutoranda em Enfermagem. Universidade Federal de São Paulo. São Paulo, Brasil. Autor de Correspondência. E-mail: fabiannesousa@hotmail.com

2 Doutora em Enfermagem. Universidade Federal do Pará. Belém, Brasil.

3 Doutora em Saúde Pública. Universidade Federal de São Paulo. São Paulo, Brasil.
Introdução: O processo de envelhecimento pode comprometer a capacidade funcional do idoso afetando sua autonomia/independência e, consequentemente a qualidade de vida. $\mathrm{O}$ objetivo desse estudo foi descrever a capacidade funcional de idosos atendidos pelo programa saúde da família da região Norte do Brasil. Materiais e Métodos: Estudo quantitativo, transversal realizado com 130 idosos residentes em áreas urbana e rural atendidos pela Saúde da Família. Avaliou-se capacidade funcional por meio do Brazilian Version of Older Multidimensional Functional Assessment Questionnaire. A análise dos dados se realizou por estatística descritiva e análise inferencial através dos testes estatísticos Qui-quadrado de Pearson e Mann-Whitney, considerando o nível de significância $\leq$ 0,05. Resultados: Houve predomínio de mulheres idosas $(57,7 \%)$, casadas $(47,7 \%)$ apresentando baixo nível de escolaridade $(59,2 \%)$. A amostra apresentou algum grau de incapacidade funcional entre os idosos da área urbana $(78,4 \%)$ e rural $(84,3 \%)$. Discussão: A capacidade funcional dos idosos deve ser preservada com ações em saúde uma vez que se caracteriza por um forte preditor da qualidade de vida. Conclusões: A boa capacidade funcional confere melhor qualidade de vida em idosos sendo condição para proporcionar um envelhecimento ativo. Avaliar à miúdo capacidade funcional dos idosos torna-se essencial para controle e manutenção do envelhecimento ativo e saudável.

Palavras chave: Idoso; Incapacidade; Enfermagem em Saúde Comunitária; Saúde da Família.

Abstract

Introduction: The aging process can compromise the functional capacity of the elderly, affecting their autonomy/ independence and, consequently, their quality of life. The aim of this study was to describe the functional capacity of the elderly cared by the family health program in the northern region of Brazil. Materials and Methods: Quantitative, cross-sectional study conducted with 130 elderly individuals living in urban and rural areas cared by Family Health. Functional capacity was evaluated through the Brazilian Version of Older Multidimensional Functional Assessment Questionnaire. Data analysis was performed through descriptive statistics and inferential analysis via Pearson and Mann-Whitney chi-square statistical tests, considering the significance level $\leq 0.05$. Results: The study showed predominance of elderly women (57.7\%), married (47.7\%) with low level of schooling $(59.2 \%)$. The sample had some degree of functional disability among the urban $(78.4 \%)$ and rural $(84.3 \%)$ elderly. Discussion: The functional capacity of the elderly should be preserved with health actions, given that it is characterized by a strong predictor of quality of life. Conclusions: Good functional capacity confers a better quality of life in the elderly, being a condition to provide active aging. Evaluating the functional capacity of the elderly is essential to control and maintain active and healthy aging.

Key words: Elderly; Disability; Community Health Nursing; Family Health.

Resumen

Introducción: El proceso de envejecimiento puede comprometer la capacidad funcional del adulto mayor afectando su autonomía/independencia y consecuentemente la calidad de vida. El objetivo de este estudio fue describir la capacidad funcional de los adultos mayores atendidos por el programa salud de la familia de la región Norte de Brasil. Materiales y Métodos: Estudio cuantitativo, transversal realizado con 130 adultos mayores residentes en áreas urbana y rural atendidos por la Salud de la Familia. Se evaluó la capacidad funcional por medido del Brazilian Version of Older Multidimensional Functional Assessment Questionnaire. El análisis de los datos se realizó por estadística descriptiva y análisis inferencial a través de las pruebas estadísticas Qui-cuadrado de Pearson y Mann-Whitney, considerando el nivel de significancia $\leq 0,05$. Resultados: Hubo predominio de mujeres adultas mayores $(57.7 \%)$, casadas $(47.7 \%)$, presentando bajo nivel de escolaridad $(59.2 \%)$. La muestra presentó algún grado de incapacidad funcional entre los adultos mayores del área urbana (78.4\%) y rural (84.3\%). Discusión: La capacidad funcional de los adultos mayores debe ser preservada con acciones en salud ya que se caracteriza por un fuerte predictor de la calidad de vida. Conclusiones: La buena capacidad funcional confiere mejor calidad de vida a los adultos mayores, siendo condición para proporcionar un envejecimiento activo. Evaluar a menudo la capacidad funcional de los adultos mayores se vuelve esencial para controlar y mantener un envejecimiento activo y saludable.

Palabras clave: Adulto Mayor; Discapacidad; Enfermería en Salud Comunitaria; Salud de la Familia.

Como citar este artigo: Sousa FJD, Gonçalves LHT, Gamba MA. Capacidade funcional de idosos atendidos pelo programa saúde da família em Benevides, Brasil. Rev Cuid. 2018; 9(2): 2135-44. http://dx.doi.org/10.15649/cuidarte.v9i2.508

(c) (i) (c) 2018 Universidad de Santander. Este es un artículo de acceso abierto, distribuido bajo los términos de la licencia Creative Commons Attribution (CC BY-NC 4.0), que permite el uso ilimitado, distribución y reproducción en cualquier medio, siempre que el autor original y la fuente sean debidamente citados. 


\section{INRODUÇÃO}

No Brasil a população idosa apresenta-se como protagonista, representando uma das transições mais aceleradas do mundo. Tal contingente impõe para os cuidados e atenção de saúde em geral de idosos, mudanças nas pessoas, na família, na sociedade, visto o processo de envelhecimento humano acarretar demandas especificas pelas modificações de ordem biopsicossociais. $\mathrm{Na}$ área biológica estão relacionados os declínios fisiológicos dos sistemas orgânicos além da diminuição da força muscular e do equilíbrio e, na área psicossocial as possibilidades de hospitalização e as demandas do processo de terminalidade dos indivíduos ${ }^{1}$.

O processo de envelhecimento deve aproximarse da funcionalidade global, essa por sua vez, é definida como a capacidade que o indivíduo possui de gerenciar sua vida, ou de cuidar de si próprio. A capacidade funcional deve encaminhar o cuidado ao idoso visando sua autonomia e independência. A autonomia pode ser definida como a capacidade do indivíduo em tomar decisões enquanto a independência, a capacidade de realizar algo pelos próprios meios ${ }^{2}$.

A capacidade funcional é um preditor importante na qualidade de vida da pessoa idosa, pode ser dividida em dois domínios: atividades físicas de vida diária (AFVD) caracterizada por ações executadas com a finalidade de autocuidado para a manutenção da sobrevivência que incluem alimentar-se, banhar-se, vestir-se e arrumar-se; e, atividades instrumentais da vida diária (AIVDs) definida por ações mais complexas que promovem interação do indivíduo com o ambiente e com a sociedade, englobam a avaliação da capacidade do idoso de como usar um telefone, fazer compras, preparar alimentos, arrumar a casa, lavar roupas, pegar ônibus, ingerir remédios e manusear as próprias finanças ${ }^{3}$.

Nesse sentido, a Política Nacional da Pessoa Idosa propõe "recuperar, manter e promover a autonomia e a independência dos indivíduos idosos, direcionando medidas coletivas e individuais de saúde para esse fim, em consonância com os princípios e diretrizes do Sistema Único de Saúde (SUS)"4.

A elaboração de atividades de promoção à saúde com vistas à preservação da capacidade funcional poderá contribuir à independência funcional assegurando um processo de envelhecimento ativo e saudável. O Programa Saúde da Família (PSF) possui papel fundamental nas atividades de promoção à saúde, é definida como a reorganização dos serviços de saúde, priorizando ações de promoção, proteção e recuperação da saúde dos indivíduos de forma contínua e integral. Nesse sentido, o PSF deve promover ações que avaliem e identifiquem a capacidade funcional dos idosos, pois políticas que não acompanham o envelhecimento das pessoas estão fadadas ao insucesso de suas ações ${ }^{5}$.

Dessa maneira, estudo realizado com idosos brasileiros residentes na área rural atendidos pelo PSF mostrou boa capacidade funcional para a realização das AFVDs e AIVDs, apesar das dificuldades geográficas enfrentadas, os profissionais de saúde promovem ações em 
saúde para proporcionar autonomia funcional aos idosos acompanhados pelo $\mathrm{PSF}^{6}$.

Nesse contexto definiu-se a questão: Como promover o envelhecimento ativo e saudável fazendo interface com a capacidade funcional de idosos usuários da ESF?

Sendo assim, o estudo teve por objetivo descrever capacidade funcional de idosos atendidos pelo programa saúde da família da região Norte do Brasil. Partiu-se da suposição de que os idosos da área urbana possuem maior independência funcional que os idosos residentes na área rural pelo maior acesso aos serviços de saúde e sociais.

\section{MATERIAIS E MÉTODOS}

Estudo quantitativo, transversal de acordo com as recomendações Strengthening the Reporting of Observational studies in Epidemiology $(\mathrm{STROBE})^{7}$, realizado em duas unidades de Saúde da Família: uma da área urbana (PSF Médice) e outra da área rural (PSF Terceira Travessa), representaram o cenário de estudo no município de Benevides, PA, Brasil. A escolha desse Município foi motivada pelo maior crescimento na longevidade dentre os municípios que compõem o estado do Pará, Região Norte. Benevides apresentou variação na taxa de envelhecimento de 3,36 para 4,46\% entre 2000 e 2010, passando da média de expectativa de vida de 66,5 anos em 2010, para 72,9 anos, em $2010^{8}$.

A amostra foi constituída de idosos usuários do PSF das áreas urbana e rural, para fins de cálculo amostral, utilizou-se a técnica de amostragem aleatória estratificada considerando o sexo e unidade de saúde? .

A população idosa de Benevides é de 4.185 idosos ${ }^{8}$, dessa forma foram selecionados, em Benevides, 65 usuários idosos de cada área urbana e rural, perfazendo 130 idosos.

Como critério de inclusão estabeleceu-se o idoso ter idade de 60 anos ou mais, acompanhados pela equipe do PSF e, exclusão ter condições de responder e/ou não responder completamente o instrumento da pesquisa, não residir na área adstrita das unidades de saúde da família.

Os dados foram coletados pela pesquisadora no período de janeiro de 2015 a janeiro de 2016. Aplicou-se um questionário sociodemográfico referente a variáveis: sexo, faixa etária, estado civil e escolaridade. Para a avaliação da autonomia e independência funcional foi utilizada a escala Brazilian version of older multidimensional functional assessment questionnaire (BOMFAQ), instrumento que determina a independência ou não nas atividades de vida diária (AVDs) como atividades física de vida diária (AFVD - deitar/ levantar da cama, comer, pentear cabelo, andar no plano, tomar banho, vestir-se, ir ao banheiro em tempo) e atividades instrumentais de vida diária (AIVD - subir um lance de escada, medicar-se na hora, andar perto de casa, fazer compras, preparar refeições, sair de condução e fazer limpeza de casa $)^{10}$. A escolha por esse instrumento foi por sua aplicação e visualização fácil. Nesse estudo foi aplicado a versão simplificada que utiliza as classificações em relação ao grau de dependência: necessita ou não de ajuda nas AVDs. Assim, a avaliação segundo os scores da escala segue abaixo: 
Escore zero $(0)$ = idoso independente funcional, que não necessitam de apoio para realizar as 14 atividades;

Escore 1 a $3=$ idoso com dependência funcional leve, que necessita de apoio para executar de 1 a 3 atividades;

Escore 4 a 6 =idoso com dependência funcional moderada, que necessitam de apoio para executar de 4 a 6 atividades,

Escore 7 ou mais = idoso com dependência funcional grave, idosos que necessita de apoio para executar 7 ou mais atividades.

Em relação a execução das atividades de vida diária o entrevistado passa a ter pontuação conforme a habilidade para a execução de cada atividade diária, dessa maneira, quanto mais alto o escore para a execução de atividades de vida diária maior é o nível de independência do idoso. Ressalta-se a capacidade da pessoa idosa de executar ou não determinada atividade e, não necessariamente executar sozinho, por exemplo, o idoso tem capacidade de cozinhar, entretanto não cozinha rotineiramente ${ }^{3}$.

A análise dos dados foi aplicada a estatística descritiva com obtenção de frequência absoluta, percentagem, média, desvio padrão, mediana, quartis mínimo e máximo para caracterização das variáveis. Análise inferencial dos dados foi processada através do software SPSS versão 20.0. Na análise inferencial bivariada, foram comparadas as atividades físicas e instrumentais de vida diária (AFVD e AIVD) entre os grupos do estudo (urbano e rural). Os testes estatísticos utilizados na comparação de variáveis categóricas foram Qui-quadrado de Pearson, enquanto que as variáveis numéricas foram empregadas o teste Mann-Whitney (distribuição não-paramétrica). Foram considerados valores estatisticamente significantes $\mathrm{p}$ valor $\leq 0,05$ para todos os testes.

O estudo foi submetido ao Comitê de Ética e Pesquisa da UNIFESP, de acordo com a Resolução 466 de 12/12/12 do Conselho Nacional de Saúde (BRASIL, 2012), obtendo aprovação - parecer n. 990.544, CAEE: 41557915.8.0000.5505. Os entrevistados que concordaram participar do estudo assinaram o Termo de consentimento livre e esclarecido (TCLE).

\section{RESULTADOS}

$\mathrm{Na}$ Tabela 1, observam-se os resultados comparativos entre as áreas urbana e rural. Nesse contexto, na área urbana a maioria era do sexo feminino em $36(55,4 \%)$, faixa etária entre 70 a 79 anos de 28 (43,1\%), com média de 73 anos de idade, casados em 24 (36,9\%), maioria com escolaridade primária incompleta em 29 (44,6\%). Enquanto na área rural a maior parte dos idosos era do sexo feminino $39(60,0 \%)$ apresentando faixa etária entre 60 a 69 anos de $47(72,3 \%)$ com idade média de 67,8 anos, casados 38 (58,5\%), escolaridade incompleta $48(73,8 \%)$. 
Tabela 1. Características sociodemográficas dos idosos das áreas urbana e rural, 2015-2016. Benevides, PA, Brasil, 2016. $(\mathrm{n}=130)$

\begin{tabular}{|c|c|c|c|c|c|c|}
\hline \multirow{2}{*}{ Variáveis } & \multicolumn{2}{|c|}{ Geral } & \multicolumn{2}{|c|}{ Área Urbana } & \multicolumn{2}{|c|}{ Área Rural } \\
\hline & N (130) & $\%$ & $\mathbf{N}(65)$ & $\%$ & $\mathbf{N}(65)$ & $\%$ \\
\hline \multicolumn{7}{|l|}{ Sexo } \\
\hline Masculino & 55 & 42,3 & 29 & 44,6 & 26 & 40,0 \\
\hline Feminino & 75 & 57,7 & 36 & 55,4 & 39 & 60,0 \\
\hline \multicolumn{7}{|l|}{ Faixa Etária } \\
\hline $60 \dashv 69$ anos & 70 & 53,8 & 23 & 35,4 & 47 & 72,3 \\
\hline $70 \dashv 79$ anos & 37 & 28,5 & 28 & 43,1 & 9 & 13,8 \\
\hline$\geq 80$ anos & 23 & 17,7 & 14 & 21,5 & 9 & 13,8 \\
\hline Média \pm Desvio Padrão & \multicolumn{2}{|c|}{$70,5 \pm 8,9$ anos } & \multicolumn{2}{|c|}{$73,2 \pm 8,3$ anos } & \multicolumn{2}{|c|}{$67,8 \pm 8,8$ anos } \\
\hline \multicolumn{7}{|l|}{ Estado civil } \\
\hline Casado & 62 & 47,7 & 24 & 36,9 & 38 & 58,5 \\
\hline Viúvo & 42 & 32,3 & 23 & 35,4 & 19 & 29,2 \\
\hline Divorciado/Separado & 12 & 9,2 & 8 & 12,3 & 4 & 6,2 \\
\hline Solteiro & 14 & 10,8 & 10 & 15,4 & 4 & 6,2 \\
\hline \multicolumn{7}{|l|}{ Escolaridade } \\
\hline Nenhuma & 28 & 21,5 & 22 & 33,8 & 6 & 9,2 \\
\hline Primário incompleto & 77 & 59,2 & 29 & 44,6 & 48 & 73,8 \\
\hline Primário completo & 16 & 12,3 & 9 & 13,8 & 7 & 10,8 \\
\hline Ensino Médio/Superior & 9 & 6,9 & 5 & 7,7 & 4 & 6,2 \\
\hline Total & 130 & 100 & 65 & 100 & 65 & 100 \\
\hline
\end{tabular}

Fonte: Elaborado pelas autoras.

Quanto à classificação do grau de dependência Tabela 2, embora sem diferença estatística, a maioria dos idosos, em ambas as áreas urbana e rural, apresentou algum grau de dependência. 
Tabela 2. Classificação do grau de dependência pela escala BOMFAQ dos idosos das áreas urbana e rural, 2015-2016. Benevides, PA, Brasil, 2016. (n=130)

\begin{tabular}{|c|c|c|c|c|c|c|c|}
\hline \multirow{2}{*}{$\begin{array}{l}\text { Grau de } \\
\text { dependência }\end{array}$} & \multicolumn{2}{|c|}{ Ger`al } & \multicolumn{2}{|c|}{ Área Urbana } & \multicolumn{2}{|c|}{ Área Rural } & \multirow{2}{*}{$\begin{array}{c}\mathbf{P} \\
\text { Valor }\end{array}$} \\
\hline & N (130) & $\%$ & N (65) & $\%$ & $\mathbf{N}(65)$ & $\%$ & \\
\hline Independente & 24 & 18,5 & 14 & 21,5 & 10 & 15,4 & \multirow[t]{4}{*}{$0.4298 *$} \\
\hline Dependência leve & 46 & 35,4 & 23 & 35,4 & 23 & 35,4 & \\
\hline Dependência moderada & 18 & 13,8 & 6 & 9,2 & 12 & 18,5 & \\
\hline Dependência grave & 42 & 32,3 & 22 & 33,8 & 20 & 30,8 & \\
\hline
\end{tabular}

* Teste do Qui-quadrado de Pearson.

BOMFAQ: Brazilian Version of Older Multidimensional Functional Assessment Questionnaire Fonte: Elaborado pelas autoras.

$\mathrm{Na}$ Tabela 3, as atividades de vida diária dos idosos, embora sem resultado significativo, verificou-se que as atividades físicas de vida diária (AFVD) autorreferida dos entrevistados da área urbana teve média de 13 atividades executadas e as instrumentais (AIVD) média de 9 atividades executadas. Enquanto os idosos da área rural obtiveram nas AFVD média de 13 atividades realizadas e AIVD média de 10 atividades realizadas.

Tabela 3. Análise comparativa da escala de atividades de vida diária (AVDs) dos idosos das áreas urbana e rural, 2015-2016. Benevides, PA, Brasil, 2016. $(\mathrm{n}=130)$

\begin{tabular}{|c|c|c|c|c|c|c|}
\hline AVDs & Área & Média & $\begin{array}{c}\text { Desvio } \\
\text { Padrão (DP) }\end{array}$ & Mediana & Mín-Máx & $P$ valor \\
\hline \multirow[t]{3}{*}{ AFVD } & Urbana & 12,9 & $\pm 2,1$ & 14,0 & 2 a 14 & \multirow[t]{3}{*}{$0.3585^{*}$} \\
\hline & Rural & 13,4 & $\pm 0,9$ & 14,0 & 10 a 14 & \\
\hline & Geral & 13,2 & $\pm 1,7$ & 14,0 & 2 a 14 & \\
\hline \multirow[t]{3}{*}{ AIVD } & Urbana & 9,5 & $\pm 4,5$ & 12,0 & 0 a 14 & \multirow[t]{3}{*}{$0.4845^{*}$} \\
\hline & Rural & 10,3 & $\pm 3,4$ & 11,0 & 0 a 14 & \\
\hline & Geral & 9,9 & $\pm 4,0$ & 11,0 & 0 a 14 & \\
\hline
\end{tabular}

*Teste não-paramétrico de Mann-Whitney.

AVDs: Atividades de vida diária

AFVD: Atividades físicas de vida diária

AIVD: Atividades instrumentais de vida diária

Fonte: Elaborado pelas autoras. 


\section{DISCUSSÃO}

No Brasil, o acelerado crescimento da população idosa impõe desafios aos pesquisadores da área gerontológica. O perfil sociodemográfico da amostra desse estudo assemelha-se a de outros estudos no contexto brasileiro. O predomínio do sexo feminino tem corroboração de outros estudos realizados como esse do nordeste brasileiro com mais de 24.000 idosos atendidos pelo PSF $^{11}$. Outro estudo realizado no sul do Brasil com idosos acompanhados pelo PSF também demonstrou predomínio de idosas ${ }^{12}$. Pesquisadores investigam se a a prevalência maior de mulheres idosas como a "feminização da velhice" são influenciadas pelo seu comportamento, pois as idosas buscam mais os serviços de saúde e conseguem conviver com mais incapacidade funcional do que os homens ${ }^{13,14}$.

A idade média dos idosos desse estudo foi de 70,5 anos, verifica-se o aumento da longevidade que pode implicar em idosos com elevado risco para incapacidade funcional ${ }^{15}$. Estudos apontam que a faixa etária entre 70 a 79 anos possui 7 vezes maiores chances de causar dano funcional quando comparado a faixa etária de 60 a 69 anos e, ainda, que os idosos com 80 ou mais de idade apresentam 3 vezes mais chances que os idosos de 70 a $79 \operatorname{anos}^{16,17}$.

Quanto ao estado civil a maioria dos entrevistados é casada, porém a viuvez revela-se como tendência corroborando com outros estudos ${ }^{12}$. Ainda, a viuvez é um importante indicador para desenvolver a incapacidade funcional, pois pode colaborar para o isolamento dos idosos e resultando na diminuição da vontade em cuidar da própria saúde, pois o estímulo do companheiro não existe mais ${ }^{16}$. Assim, a viuvez pode revelarse em um indicador de risco de mortalidade e ao agravamento de morbidade ${ }^{14}$.

A escolaridade dos idosos é considerada baixa, estudos apontam que o analfabetismo na população idosa é alto, podendo ser justificado pela dificuldade de acesso às escolas ${ }^{18}$. Corrobora ainda com esse resultado, estudos com idosos do nordeste brasileiro que apresentaram escolaridade baixa. De acordo com essa literatura, a partir da década de 50, existia dificuldade ao acesso à educação principalmente pelas mulheres ${ }^{14,19}$.

Na análise comparativa, o estudo revelou minoritariamente que os idosos da área urbana são independentes funcionalmente em relação aos da área rural; porém é relevante destacar que o resultado em sua maioria mostra dependência funcional leve com tendência à grave em ambas as áreas. Nesse caso estudos apontam que a capacidade funcional é uma importante ferramenta para a elaboração de políticas públicas para a melhora da qualidade de vida ${ }^{12}$. O aumento da idade pode estar associado com o grau de dependência dos $\operatorname{idosos}^{20,21}$.

A capacidade funcional pode ser definida pela realização das AFVD e AIVD suficientes para uma vida com autonomia e independência funcional. Para o idoso, a realização dessas atividades de vida diária aparece como algo presente e necessário para a sua sobrevivência, mantendo-o participativo na gestão e nos cuidados com a própria saúde ${ }^{14}$. 
Um aspecto relevante, como resultado nesse estudo, a longevidade apresenta-se em ambas as áreas, assim, corrobora com a afirmação de que a idade avançada exerce influência relevante na qualidade de vida, no tocante ao desempenho das atividades funcionais ${ }^{22}$.

A Organização mundial de saúde recomenda que para o envelhecimento seja ativo, é necessário possibilitar que os indivíduos entendam o seu potencial para o bem-estar físico, social e mental no desempenho de suas atividades cotidianas. Essa capacidade é alcançada também com intervenções que criam ambientes de apoio e promovam opções saudáveis em todos os estágios da vida ${ }^{16}$. Dessa forma, é importante ressaltar que a ESF deve realizar o monitoramento da capacidade funcional visando à manutenção da autonomia e independência do idoso gerando medidas que promovam a realização das AFVD e $\operatorname{AIVD}^{14}$.

No que concerne às atividades de vida diária, de um modo geral foi possível encontrar que os idosos da área urbana e da área rural possuem similaridades no escore para AFVD e AIVD; apesar de estudos revelarem que existe um aumento da concentração de idosos no ambiente urbano, sobretudo por permitir melhores condições de saúde e acesso aos serviços de saúde $^{23,24}$. Esse resultado aponta que os idosos possuem dificuldades na realização das AIVD possivelmente devido a tendência do aumento da longevidade bem como a viuvez, ou seja, aumento dos idosos septuagenários e/ou octogenários viúvos.
Foi possível constatar durante nosso estudo, que as atividades desenvolvidas pelos idosos da área rural como, por exemplo, na participação efetiva de atividades agrícolas dentre essas, o cultivo de flores, promovam um envelhecimento ativo e saudável ${ }^{23}$. Corrobora, ainda desse resultado, estudo realizado com idosos residentes da área rural que afirma que os idosos residentes na área rural possuem melhores condições de vida quando comparados com a área urbana ${ }^{19,25,26}$.

Como limitação desse estudo, o fato de ser transversal interfere no acompanhamento da capacidade funcional dos idosos, tornase necessário a realização de mais estudos, possivelmente longitudinais que propiciem o conhecimento e controle da capacidade funcional dos idosos acompanhados pelo Programa Saúde da Família.

\section{CONCLUSÕES}

O estudo permitiu caracterizar a população idosa inclusive sua capacidade funcional. Mulheres idosas, com média de 70,5 anos, casadas, porém com tendência à viuvez, escolaridade baixa, apresentando algum grau de dependência funcional na maioria dos idosos, independentemente da área urbana ou rural.

Os idosos daárea urbana possuem "discretamente" melhor escore para a capacidade funcional preservada, supondo-se que as equipes de saúde do PSF ofereçam melhor acesso aos serviços em ambiente urbano. Possivelmente o acesso aos serviços de saúde pelos idosos da área rural sejam dificultados pela área geográfica.

Entende-se por esse resultado que a capacidade funcional não foi determinada pela área onde o 
idoso reside, mas por atividades que possam a vir desenvolver. Destaca-se no estudo à similaridade dos resultados na realização das AFVD e AIVD dos idosos entre as áreas: urbana e rural.

A boa capacidade funcional desenvolve fator importante que confere melhor qualidade de vida aos idosos sendo uma condição relevante para proporcionar um envelhecimento ativo. Avaliar à miúdo capacidade funcional dos idosos torna-se essencial para controle e manutenção do envelhecimento ativo e saudável.

\section{Agradecimentos: À CAPES (Coordenação de Aperfeiçoamento de Pessoal de nível Superior) pela concessão da bolsa de pesquisa.}

Conflitos de interesse: Os autores declaram que não há conflito de interesses.

\section{REFERENCIAS}

1. Brito KQD, Menezes TN, Olinda RA. Functional disability and socioeconomic and demographic factors in elderly. Rev. Bras. Enferm. 2015; 68(4): 633-41. http://dx.doi.org/10.1590/0034-7167.2015680409i

2. Santos GLA, Santana RF, Broca PV. Capacidade de execução das atividades instrumentais de vida diária em idosos: Etnoenfermagem. Esc. Anna Nery. 2016; 20(3): e20160064. http://dx.doi.org/10.5935/1414-8145.20160064

3. Ramos LR, Andreoni S, Coelho-Filho JM, Lima-Costa MF, Matos DL, Rebouças M. Perguntas mínimas para rastrear dependência em atividades da vida diária em idosos. Rev. Saúde Pública. 2013; 47(3): 506-13. http://dx.doi.org/10.1590/S0034-8910.2013047004325

4. Brasil. Ministério da Saúde. Portaria $n^{\circ}$. 2.528, de outubro de 2006. Política Nacional de Saúde da Pessoa Idosa. Diário Oficial da União 20 de outubro de2006; Seção 1.

http://bvsms.saude.gov.br/bvs/saudelegis/gm/2006/ prt2528 $19 \quad 10 \quad 2006 . h t m l$

5. Wendt $\bar{C}$ JK, Aires M, Paz AA, Fengler FL, Paskulin LMG. Elderly families of South of Brazil in the Health Strategy. Rev Bras Enferm. 2015; 68(3): 350-7. http://dx.doi.org/10.1590/0034-7167.2015680305i
6. Pinto AH, Lange C, Pastore CA, Llano de PMP, Castro DP, Santos F. Functional capacity to perform activities of daily living among older persons living in rural areas registered in the Family Health Strategy. Ciência \& Saúde Coletiva. 2016; 21(11):3545-55. https://doi. org/10.1590/1413-812320152111.22182015

7. EIm EV, Altman DG, Egger M, Pocock SJ, Gotzsche PC, Vandenbroucke JP. The Strengthening the Reporting of Observational Studies in Epidemiology (STROBE) statement: guidelines for reporting observational studies. The Lancet. 2007; 370 (9596):1453-57. https://doi.org/10.1016/S0140-6736(07)61602-X

8. Instituto Brasileiro de Geografia e Estatística (IBGE). Censo Demográfico. Rio de Janeiro: IBGE; 2016.

9. Bolfarine H, Sandoval MC. Introdução à Inferência Estatística. Rio de Janeiro: SBM; 2011.

10. Ramos LR, Rosa TE da C, Oliveira ZM, Medina MCG, Santos FRG. Perfil do idoso em área metropolitana na região sudeste do Brasil: resultados de inquérito domiciliar. Rev. Saúde Pública. 1993; 27(2): 87-94. http://dx.doi.org/10.1590/S0034-89101993000200003

11. Barbosa KTF, Costa K, Macêdo NF, Pontes MLF, Batista PSS, Oliveira FMRL, et al. Envelhecimento e vulnerabilidade individual: um panorama dos idosos vinculados à estratégia saúde da família. Texto contexto enferm. 2017; 26(2): e2700015.

http://dx.doi.org/10.1590/0104-07072017002700015

12. Gavasso WC, Beltrame V. Capacidade funcional e morbidades referidas: uma análise comparativa em idosos. Rev. bras. geriatr. gerontol. 2017; 20(3): 398-08. http://dx.doi.org/10.1590/1981-22562017020.160080

13. Duarte MCS, Fernandes MGM, Rodrigues RAP, Nóbrega MML. Prevalência e fatores sócio econômicos associados à fragilidade em mulheres idosas. Rev Bras Enferm. 2013; 66(6): 901-6.

https://doi.org/10.1590/S0034-71672013000600014

14. Ferreira AP. Capacity and performance for the realization of basic activities of daily living (basic and instrumental) in elder dependents. Rev. baiana saúde pública. 2015; 39(1). http://dx.doi.org/10.5327/Z0100-0233-2015390100004

15. Britto TA, Fernandes MH, Coqueiro RS, Jesus CS. Quedas e capacidade funcional em idosos longevos residentes em comunidade. Texto \& Contexto Enferm. 2013; 22(1): 43-51.

16. Lourenço TM, Lenardt MH, Kletemberg DF, Seima MD, Tallmann AEC, Neu D, K M. Capacidade funcional no idoso longevo: uma revisão integrativa. Rev. Gaúcha Enfer. 2012; 33(2): 176-85. http://dx.doi.org/10.1590/S1983-14472012000200025

17. Quijada PDS, Fernandes PA, Ramos SB, Santos BMO. Qualidade de vida relacionada à saúde de pacientes com câncer de próstata. Rev Cuid. 2017; 8(3): 1826-38. http://dx.doi.org/10.15649/cuidarte.v8i3.436

18. Pletsch LE, Dallepiane, LB, Kirchner RM, Anacleto SLA, Silva P, FK, J, Gopinger E, Martinazzo CJ. Perfil sociodemográfico e de hábitos de vida da população idosa de um município da região norte do Rio Grande do Sul, Brasil. Rev Bras de Geriat e Gerontol. 2014;17(2): 30314. https://doi.org/10.1590/S1809-98232014000200008 
19. Tavares DMS, Fachinelli AMP, Dias FA, Bolina AF,Paiva MM. Preditores da qualidade de vida de idosos urbanos e rurais. Rev Baiana Enferm. 2015; 29(4): 361-71. https://doi.org/10.18471/rbe.v29i4.13359

20. Feng MA, McMillan DT, Crowell K, Muss H, Nielsen ME, Smith AB. Geriatric assessment in surgical oncology: A systematic review. J Surg Res. 2015; 193(1): 265-72. https://doi.org/10.1016/j.jss.2014.07.004

21. Wang H, Chen K, Pan Y, Jing F, Liu H. Associations and impact factors between living arrangements and functional disability among older Chinese adults. PLoS One. 2013; 8(1): e53879. https://doi.org/10.1371/journal.pone.0053879

22. Marinho CLA, Oliveira JF, Borges JES, Fernandes FECV, Silva RS. Associação entre características sociodemográficas e qualidade de vida de pacientes renais crônicos em hemodiálise. Rev Cuid. 2018; 9(1): 2017-29. http://dx.doi.org/10.15649/cuidarte.v9i1.483

23. Ribeiro CG, Ferretti F, Sá CA. Qualidade de vida em função do nível de atividade física em idosos urbanos e rurais. Rev. bras. geriatr. gerontol. 2017; 20(3): 330-9. http://dx.doi.org/10.1590/1981-22562017020.160110.

24. Bautista LM, Arias MF, Carreño ZO. Percepción de los familiares de pacientes críticos hospitalizados respecto a la comunicación y apoyo emocional. Rev Cuid. 2016; 7(2): 1297-1309. http://dx.doi.org/10.15649/cuidarte.v7i2.330

25. Ferreira OGL, Maciel SC, Costa SMG, Silva AO, Moreira MASP. Envelhecimento ativo e sua relação com a independência funcional. Texto Contexto Enferm. 2012; 21(3). http://www.scielo.br/pdf/tce/v21n3/v21n3a04

26. Tavares DMS, Ferreira Pollyana CS, Dias FA, Oliveira PB. Characterization and spatial distribution of male octogenarians. Rev enferm. 2016; 22(4): 558-64. 